\title{
"Of Mastery and Weariness": Qualitative Evaluation of the Lecture-RLE Series Scheduling Scheme
}

\author{
Martinez, Rudolf Cymorr Kirby \\ rmartinez@sanbeda.edu.ph \\ San Beda University - College of Nursing \\ Arellano University - Graduate School of Nursing
}

\begin{abstract}
This paper explored the experiences of students on the Lecture-RLE Series Scheduling Scheme (LRSS) both as an evaluation of the program and as a basis for enhancement. Grounded on the interpretive paradigm, forty two (42) participants were recruited to partake in this study. Further, reflection papers from the participants serve as the narratives of the study and where then reflectively analyze via the process of thematization.

After the three (3) level of reflective analysis, the following themes were identified: (1) Dedication: Focused Learning; (2) Mastery of Professional Qualities; (3) Setbacks: Stress \& Weariness. The themes shows that in general, the overall experience of the participants was affirmative and optimistic claiming that LRSS promotes a conducive learning environment wherein they felt "less pressured" to perform task. Further the participants felt that the LRSS provides them with a mean to fully realize the development of their professional qualities, skills and attitude of a "real" nurse since it mirrors the realities of an "on-the-job-training". Lastly, the identified setbacks of the LRSS are two-folds: stress and a sense of weariness, largely attribute to the consecutive days of duty schedules as well as a perceived monotony of activities in the area of exposure.

Insights from this study suggest that there is a need to update and upgrade strategies and methodologies of teaching implemented in the clinical area to provide varied approaches to learning skills as well as limit the perception of monotony among student clinicians. Further research that dwells on the (1) nature of stress, (2) the patterns of coping and adjustment among student clinicians is also implied.
\end{abstract}

Keywords: Qualitative Research, Nursing Education, Related Learning Experience

Martinez, R. C. K. P. (2019). "Of Mastery and Weariness": Qualitative Evaluation of the LectureRLE Series Scheduling Scheme. SocArXiv. Doi: 10.31235/osf.io/nb8pa 


\section{CONTEXT OF THE STUDY}

The San Beda University College of Nursing has adopted an unorthodox method in plotting its didactic and related learning experiences. Rather than the traditional method of having related learning experience rotations in concurrence with classroom lectures, the College has crafted a scheme wherein students undergo 12 weeks of pure classroom lectures, followed by 6 weeks of uninterrupted clinical exposures. The consistent nurse licensure examination performance of graduates, the positive feedback from affiliate institution, the students and clinical instructors plus the relatively low occurrence of errors committed by students have encouraged the continued adoption of the scheme.

However, a recent visit by the Philippine Accrediting Association of Schools, Colleges and Universities (PAASCU) has raised some questions on the effectiveness of the "Lecture-RLE Series Scheduling" (LRSS) scheme used by the College thus in order to address the concern, the College has commissioned the conduct of this study. This study specifically employed the qualitative approach to deeply understand and investigate the experiences of students in a contextualized manner.

This preliminary evaluation of the "Lecture-RLE Series" teaching scheme in the San Beda College of Nursing specifically aims to explore the insights of the students who have undergone its teaching methodology. It limits itself to evaluating the LRSS scheme through the experiences of the students and not through other variables thus the implications arising from the insights generated by this study can only be ascertain within the context of the participants' reality and may not be transferrable to other select group of student.

The researchers are optimistic that this scientific inquiry into a novel teaching scheme in nursing instruction is a step towards the future of nursing education since as Giden and Brady (2007) puts it: there is a dire need for reforms and innovation. 


\section{METHODOLOGY}

This research is grounded on the qualitative paradigm to understand the experiences of select students in the lecture-RLE series scheduling (LRSS) scheme. Specifically the adopted approach developed by Martinez (2013) was utilized as the method of analysis rooted on the process of thematization. Forty-two (42) participants, composed of 24 junior and 18 senior students, the total current population of the College, were recruited to partake in this study. They were purposively selected since they have undergone the "Lecture-RLE Series" teaching methodology for at least a year ( 2 semesters). The participants were instructed to write a reflection paper on their experience of the LRSS scheme which serves as the "raw data" of the study.

The following process describes and summarizes how the experiences were reflectively analysed in this study. Specifically, it shows the process of reflective analysis of the experiences, together with its concurrent level of reflection, essential steps, ways of enriching the experiences and their outcomes (Figure 1).

The yellow shaded boxes shows the level of reflections that was utilized in this study, opposite of which, blue shaded boxes, are the essential steps in the reflective analysis related with each level. In addition to that, the pink shaded boxes represent the ways on how the experiences was enriched, validated and countervalidated, by the participants' experiences. The green shaded boxes represent the outcomes of each essential steps as well as each level of reflections.

The following are the essential steps in the reflective analysis of the participants' experiences

1. Written reflections from the participants were gathered after which they were encoded verbatim. This represents the first essential steps termed as the "gathering of experiences". Thereafter, the encoded reflections were given back to participants to validate their content which then resulted in the narrative, a validated account of the participant's experiences and will be thus considered as the "raw data".

2. After the transcription, the researchers then identified the essential meanings found in these narratives via identifying their thought markers. As a result of this first reflection, the enhanced and validated thought element of the experiences was produced. During this phase, member checking of the thought elements were 
performed both to maintain the rigor of the study as well as ascertain its credibility. This was done by first separately extracting the thought elements by the individual researchers and then convening the research team to discuss and compare notes on the process. The end goal is to produce a single version of the narratives with thought elements agreed upon by the research team.

3. Next, after the meaning units of the combined experiences were explicated, the researcher then reflected on these thought elements to give birth to themes, representing the second level of reflection, the thematic interlace. The resulting themes were again validated and counter validated by the participants through an interview which then results in the enriched and validated themes.

4. To embellish grounding to the significant insights gathered from the process of reflective analysis, reflective resonance was done by situating the resulting themes from literatures. This process, paralleling the related literature review done in a quantitative paradigm, will neither affirm nor negate the insights formed from the reflection but merely add a lens by which the result can be appreciated, following the philosophical underpinning of a contextual understanding based on the tradition of the qualitative philosophy. This constitutes the 2 rd level of reflection, the thematic embodiment.

The process of validation and counter validation was done by presenting the significant outcomes of each step in the process of reflective analysis to the participants for resonance. It is only when they have agreed that the outcomes mirrors their narratives shall the researchers continue to the next level of reflective analysis. This continuous process of validation and counter validation ascertain that great

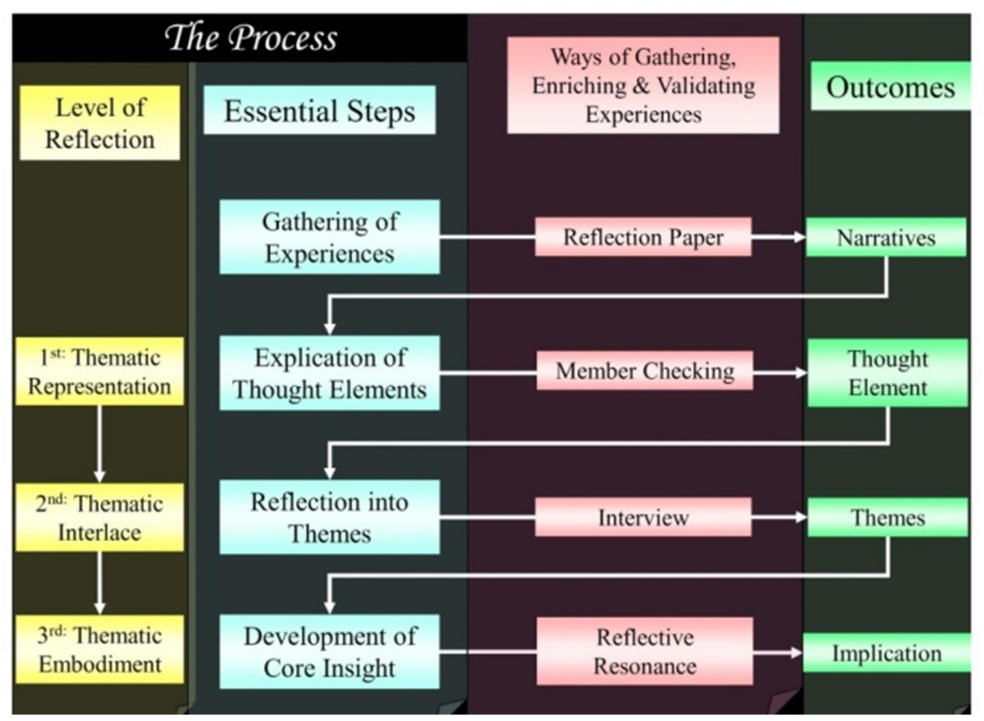

Martinez. R. C. K. P. (2019). "Of Masterv and Weariness": Oualitative Evaluation of the Lecture- 
Figure 1: The research process developed by Martinez (2013) showing the process of reflective analysis of the experiences, together with its concurrent level of reflection, essential steps, ways of enriching the experiences and their outcomes

emphasis is placed on the primacy of the participants' narratives as well as ground the study on the tenet that understanding is a co-created reality between the researchers and the participants.

\section{MAINTAINING ETHICAL STANDARDS}

This study upholds ethical standards throughout its process of inquiry. Respect for human dignity was upheld with the highest standard all throughout the study. This said standard will be reflected in this paper by the following methods.

1. Approval of the Internal Review Board was solicited prior to beginning the inquiry

2. Informed consent was secured from the prospective participants after explaining the objectives of the study, the risks involved, the extent of their participation, assurance of the confidentiality of their identities and/or any identification that can be alluded to the participants on the information that they provide. Voluntary withdrawal anytime along the conduct of the study may be done by the participants without any question, explanation or repercussion was also be explained together with the possibility that the research might be published in a local or international journal.

3. Anonymity of the participants was maintained by providing number codes instead of their true identity as well as making their narratives (raw data) only available to the research team and not including it in any part of the final manuscript.

4. Safe keeping of information in the form of notes was facilitated by securing it in a locked cabinet and only the research team held the key to such.

5. Going back and forth to the participants for the ongoing process of validation and counter validation and final approval of the content of the study and

6. Destroying the hard data gathered through burning, a year after finishing the study 


\section{RESULTS AND DISCUSSION}

Narratives from 42 participants were synthesized into 126 thought elements and these thought elements were further explicated to form 13 clusters containing essentially parallel elements. The following clusters were thus identified: Setback/disadvantage; efficient and comfortable; focused; analytical and organized, helpful; appreciative of lecture; preparedness; confident; comparison to other methods; mastery; professional nurse; fear. It must be noted however that these clusters are not the end in itself but a mean to further understand the themes, the significant insights, of the study. Following the process described above, three (3) themes reflective of the participants experiences were thus culled out and are as follows: Dedication: Focused Learning; Mastery of Professional Qualities; Setbacks: Stress \&Weariness

\section{Themes}

\section{Dedication: Focused Learning}

This theme explores the students experience of the L-R series as promoting a conducive learning environment wherein the students felt "less pressured" to perform task, by giving priority to fully mastering theory prior to practice.

For them, one of advantages of the L-R series is that it focuses first on understanding the theoretical basis before the actual performance/ exposure in the area. It enables them to fully grasp the topic by zeroing in on learning the principle first without the burden of preparing for the duty (RLE) unlike the traditional model. This was perceived by the students as less stressful thus facilitating the learning process wherein the mastery of the concepts was claimed to be more fluid and dynamic. A student shares that

"I do not need to prepare for our duty the same time as we prepare for our lecture"

Moreover, some students who have undergone the traditional method verbalized that the traditional method is physically taxing since time as well as their focus is divided both for their lecture and RLE preparations. 
With much focus given by the students to classroom instructions, students verbalized that they appreciate more the lectures, can focus more on studying and maximize the time to acquire knowledge prior to the actual exposure in the area. Having a pure classroom instruction first let the student feel that they are better guided in learning, motivated in studying and prepared in their future exposure in the clinical area.

When it comes to the learning process, for the students, grasping mastery of the concept, therefore, is given priority than acquiring the ability to multitask.

\section{Mastery of Professional Qualities}

This theme explicates the students' appreciation of the Lecture-RLE series as a mean to fully realize the development of their professional qualities equip with the proper knowledge, skills and attitude.

Because the Lecture-RLE series was seen by the student as a mean to fully prepare them with the needed concepts, they verbalized that they feel more confident in the performance of their task during their RLE exposure. They further claimed that the scheme enabled them to perform their clinical task with relative ease since all of the needed concepts were discussed prior to their clinical exposure. They verbalized that because they already know the concepts, it is will be like "just a simple review" when they try to recall things as opposed to learning the concept without any prior lecture. It seems that for the student, their confidence is built upon the knowledge of their perceived mastery of the concepts prior to their exposure.

In the Lecture-RLE series, students are exposed in the clinical area 4-5x a week with minimal lecture class. For the student, this scheme let them feel as if "they are like a true nurse" in the area since their shifting schedule is almost identical to staff nurses enabling them to acculturate more to the routine, nuisance and culture of the clinical area. One student verbalize

"You really feel that you are a nurse and you will be ready to become a professional nurse in the future since you can see what they really do"

The students also verbalized that the scheme enables them to know their patient more since they are exposed at bedside on a relatively longer period of time. 
This facilitates the development of rapport and trust with the students and their patients that lets the students render care on a more personal level since they "see the patient not only as a patient but also as a human being". Within this context, the student felt more fulfilled, and satisfied with the care that they give to their patients. Working together with the staff nurses on their duty hour that are almost parallel, the students verbalized that they appreciate more their part as a member of the health care team and not just merely as a student. The student also verbalized that this enables them to be more familiar with the members of the health care team and vice versa thus facilitating rapport among them making the work environment "less stressful".

For the students, the Lecture-RLE series allows the realization and development both of their professional qualities and human character.

\section{Setbacks: Stress and Weariness}

This theme elucidate on the participants notion of the LRSS scheme's setback, namely stress and the perceived sense of weariness during the duration of the program. These two concepts resonate among the participants during the process of validation and counter validation as the main impediment of the LRSS scheme.

The participants claimed that the scheme's consecutive day's schedule, 5 for juniors and 4 for seniors, give them "stress" at times especially during the first few days. Though the participants are aware that the stress for the first few days could be attributed to their process of adaptation of the routines of the hospital setting, some verbalized that this stress continued well beyond the adaptation stage. It is interesting to note however that the LRSS scheme only seems to "add up" to their stress and not the root cause of it. One participant's verbalization that the consecutive days of duty "nakakadagdag sa stress (Adds up to stress)" echoed well among the other participants. Asked if the duty per se caused them stress, the participants would answer "di naman, yung schedule lang (not really, only the schedule)"

Another setback that the participants identified is the feeling of weariness from having monotonous activities during the clinical exposure. The participants verbalized that though mastery and familiarity is built through repetitive activities, sometimes it bores them. One participant explains 
"Minsan nakakasawa yung paulit-ulit yung ginagawa sa ward

(Sometimes it becomes boring when you do the same activities in the ward)"

For the participants, the duty scheduling and the nature of activities in the LRSS scheme contribute to its disadvantage as it add up to their stress and is perceived as repetitive and monotonous thus seen as "boring".

\section{Core Insight}

The themes shows that in general, the overall experience of the participants was affirmative and optimistic claiming that LRSS promotes a conducive learning environment wherein they felt "less pressured" to perform task, by giving priority to fully master theory prior to the hands-on practice. Further the participants felt that the LRSS provides them with a mean to fully realize the development of their professional qualities and equip them with the proper knowledge, skills and attitude of a "real" nurse since it is more grounded on the day to day realities of an "on-the-jobtraining" (OJT). Lastly, for the participants, the identified setbacks of the LRSS are two-folds: stress and a sense of weariness. The participants largely attribute these to their consecutive days of duty schedules as well as a perceived monotony of activities in the area of exposure.

\section{Thematic Resonance}

This section provide context from existing literature to further provide another layer of understanding the resulting insights of this study. It does not aim to compare and contrast but rather to merely situate the insights from available foreign studies.

Having limited distractions, in the form of preparations for clinical exposure, during the lecture days enable the participants to focus more on understanding the concepts and theories facilitating the learning process. Focusing on a limited task one at a time demands less physical and psychological effort thus there is a fluid transfer of knowledge. Moreover, for the participants, focusing first on the lecture enables them to feel at ease and be more motivated to learn and prepare for the hospital exposure. This minimizes anxiety as a result of feeling incompetent, echoing the result of the study done by Sharif \& Masoumi (2005). 
Feeling less pressured in the clinical setting was seen by the participants as a contributing factor in their mastery of skills. This confidence was seemingly developed during the intensive lecture prior to their exposure in the area. This was parallel to the result of the study done by Kim et al (2014) and Shepard (2009) which posts it that the degree of confidence is related to the perceived competence in skills. Further, with a developed confidence and an intensive clinical exposure, the participants verbalized that they imbibed more a sense of being a professional nurse.

Being exposed in the area for a longer period of time enables the participants to develop their interpersonal skills, such as rapport building among patient and their family. This was parallel to the studies which noted that students' confidence (Wieland et al, 2007) and satisfaction (Warne et al, 2010) increase with a longer exposure to their area as they appreciate the whole individual nursing process over a longer period of time. Another aspect of the LRSS scheme that the student found positive is the paralleling of their duty schedule to that of the staff nurses. This enables them to "be in the shoe" of a professional nurse and have a better appreciation of the profession building competence among the participants. Greater amount of interaction with the staff nurse was seen as developing a greater degree of competence among student as was shown by the study of Haitana \& Bland (2011); Kim (2007); Kim et al (2004).

The parallel duty hours of the participants and the staff nurse also contributed to students' sense of fulfilment and satisfaction with the care they rendered since they feel that they are part of the health care team. This was also reflected in the study done by Warne et al (2010) which emphasize that the longer interaction with the staff nurse enables the student to recognize the crucial element of the caring relationship. Neary (1997) also found the same insight in this study and concluded that providing a sense of belonging by being part of the caring team alleviate fear and insecurities among students.

One setback of the LRSS scheme is credited to stress attributed to the consecutive duty days within the week. Gibbon et al (2011) similarly found out that much of the stress of nursing student can be attributed to "placement-related demands" such as duty schedules. Another perceived setback is the sense of weariness among the participants largely ascribed to monotonous, repetitive clinical activities. Papastavrou (2009) found a similar pattern in his study which points out that the 
learning environment was seen by the participants as contributing to their "premise of learning".

\section{IMPLICATIONS OF THE STUDY}

The core of a qualitative inquiry is a deeper understanding of the phenomenon under exploration thus following its philosophical root; it does not claim to generalize the insights gathered from the process of reflection nor aim to give recommendations based on the reflected realities gleaned from this approach. Instead, the insights, vis-àvis themes, are utilized to make suggestive implications drawn solely from the phenomenon, confine within the context of this study. After careful reflection on the resulting insights, vis-à-vis themes, of the study, the following implications are thus put forth.

1. The Lecture -RLE series is generally favoured by the students as it promotes both mastery of theoretical knowledge and clinical skills by providing an environment that is less stressful

2. Though it promotes familiarity with the clinical setting, and rapport with the members of the health care team, and their patients, some students verbalize exhaustion at some point during the clinical exposure. It may be implied that a break in the RLE duty schedule, either through a day off or a classroom lecture day, might be warranted.

3. Insights from this study suggest that there is a need to update and upgrade strategies and methodologies of teaching implemented in the clinical area to provide varied approaches to learning skills as well as limit the perception of monotony among student clinicians.

4. Since stress was an incidental reason for the perceived setback of the LRSS scheme, further research that dwells on the nature of stress and patterns of coping and adjustment among student clinicians is also implied.

5. Further research comparing the traditional scheme and the Lecture-RLE scheme in terms of students acquired knowledge and skills, level of preparedness for RLE exposure as well as student's level of stress is needed. 


\section{References}

Gibbons, C., Dempster, M., Moutray, M. (2011) Stress, coping and satisfaction in nursing students. Journal of Advanced Nursing, 67(3), 621-632.

Giddens, J., Brady, D. (2007). Rescuing nursing education from content saturation: the case for a concept-based curriculum. Journal of Nursing Education, 46(2), 65-69.

Haitana, J., \& Bland, M. (2011).Building relationships: The key to preceptoring nursing students. Nursing Practice in New Zealand, 27(1), 4-12.

Kim, K. (2007). Perceived competency of senior nursing students after their clinical preceptorship programs. Journal of Professional Nursing, 23(6), 369-375.

Kim, K., Lee, A., Eudey, L., Dea, M. (2014).Improving clinical competence and confidence of senior nursing students through clinical preceptorship. International Journal of Nursing, 1(2), 184-209.

Martinez, R. (2013). "Masked": The lives of adolescents undergoing chemotherapy. Philippine Journal of Nursing, 83(2), 9-18.

Neary, M.(1997). Project 2000 students' survival kit: a return to the practical room. Nurse Education Today, 17(1): 46-52.

Papastavrou, E., Lambrinou, E., Tsangari, H., Saarikoski, M., Leino-Kilpi, H. (2009). Student nurses experience of learning in the clinical environment. Nurse Education in Practice, 10(3), 176-182.

Sellek, T. (1982).Satisfying and anxiety creating incidents for nursing students. Nursing Times, 78(35): 137-140.

Sharif, F., Masoumi, S. (2005). A qualitative study of nursing student experiences of clinical practice. BMC Nursing.4(6).doi:10.1186/1472-6955-4-6

Shepard, L. (2009). Examining the effectiveness of a preceptorship on clinical competence for senior nursing students in a baccalaureate program.. Dissertations Abstracts International, 71(3), DAIB.(UMI No. AAT 3396353)

Warne, T., Johansson, UB., Papastavrou. E,,Tichelaar, E., Tomietto, M., Van den Bossche, K., Moreno, MF., Saarikoski, M. (2010). An exploration of the clinical learning experience of nursing students in nine European countries. Nurse Education Today. 30(8), 809-815.

Wieland, D., Altmiller, G., Dorr, M., \& Wolf, Z. (2007).Clinical transition baccalaureate nursing students during preceptorship, pre-graduation practicum. Nursing Education Perspective, 28(6), 315-321. 